\title{
Establishing an Optimization Model for Sewer System Layout with Applied Genetic Algorithm
}

\author{
H. T. Weng* and S. L. Liaw \\ Graduate Institute of Environmental Engineering, National Central University, Chungli 32054, Taiwan
}

\begin{abstract}
In this study, a genetic algorithm (GA) is first used to establish a combinatorial optimization model, called the Sewer System Optimization Model for Layout \& Hydraulics (GA/SSOM/LH), to find an optimal design for a real urban sewer system. The problems of "network layout" and "hydraulic design" optimization are considered simultaneously. The modeling concept is to combine the fundamental principles of the GA, to the generation of possible network layouts as well as to develop a "hydraulic design" optimization module, the Sewerage System Optimization model (SSOM), which can find the best sewer system layout by checking the overall least-cost hydraulic design of several possible alternate network layouts. SSOM is a 0-1 Mixed Integer Programming (MIP) in which a traditional algorithm, the Bounded Implicit Enumeration (BIE) is applied to determine the optimal size and slope for each. Unlike the BIE algorithm, 'one chromosome' in the GA evolution is coded to represent 'one system layout' parameter. Specific coding strings on 'parameters' are operated directly and are more robust when combined with the SSOM module. Hence the GA can evolve quickly generating an optimized system layout and ensuring a solution closer to the global optimum in a 'fast' manner. Finally, a case study was conducted on a 73-node project to verify the optimal system layout as generated by the GA/SSOM/LH model.
\end{abstract}

Keywords: Bounded Implicit Enumeration, Genetic Algorithm, hydraulic design, N-P Complete problem, system layout, 0-1 Mixed Integer Programming

\section{Introduction}

In order to reach the goal of a higher household connection rate to sewer systems in Taiwan, the government is going to allocate more funding to urban sewerage construction. Thus cost-effectiveness analysis becomes an important issue for the optimal design of new sewer systems. A sewer system uses gravity to collect and transport sewage from a house to sewage treatment plants through a network of hydraulically designed sewer pipes, connecting the sewer pipe-network, manholes, pumping stations and other related appurtenances. The design of a sewer system may be divided into two phases: (1) the selection of the network layout; and (2) the hydraulic design of the sewer pipes for the selected layout (this require the determination of the discharge rates, the pipe sizes, the slopes, and invert elevations) (Tekeli and Belkaya, 1986). The consideration of the problem of the optimality of the two design phases would certainly head to an optimal "network layout" with the least-cost "hydraulic design" of a sewer system, which will really achieve the optimal sewer "system layout" for sewer design problems. Since simultaneous consideration of optimal "network layout" and "hydraulic design" phases is too complex, each phase is usually dealt with separately. This approach may reduce the complexity of the design procedure, but the final design obtained may not be the most optimum when cost-effectiveness is the concern.

\footnotetext{
* Corresponding author: tyng@ms14.hinet.net
}

In practice, a design is produced by manually generating an adapted network layout that will meet the needs of the population to be served, and fit the street layout and the local topography of the planning area. Then a hydraulic design is made and the pipe sizes and excavation depths for a specific layout are found. Obviously, the result is limited by the engineer's experience and intuition and only a very small number of the alternatives can be evaluated. The final design is sometimes deficient and there is no guarantee that it is the best design. The finding of an optimal sewer "system layout" is surely not an easy job for engineers. The difficulty is mainly due to two important factors: the complexity of the system's environment and the huge number of possible design alternatives. Particularly in the large networks necessary for urban sewer system, manual calculation limits the evaluation of alternatives. This has inspired many computerized optimization model studies. Various algorithms and mathematical models have been applied with the aid of high-speed electronic computer system, which substituting traditional manual time-consuming calculation to get cost-effective design.

In the past three decades, efforts have been made to develop models to find the optimal hydraulic designs for the construction of new-branched sewer systems. Optimal design problems are larger than traditional approaches in terms of effectiveness and efficiency. However, the available models for generating optimal network layouts and for completing hydraulic designs require extensive computations necessitating excessive execution time. Consequently, while most mod- 
els have been developed for the optimization of "hydraulic designs” (Merrit and Bogan, 1973; Dajani and Hasit, 1974; Mays and Yen, 1975; Wen and Kuo, 1982; Gupta et al., 1983; Shih, 1983; Orth and Hsu, 1984; Orth, 1986; Liaw and Lin, 1990; Liaw, 1991; Agbenowosi,1995; Charalambous and Elimam, 1997; Greene et al., 1999; Swamee, 2001; Weng and Liaw, 2003); only a few models have been developed for the optimization "system layouts". In this study, we focus on the model for the optimization of 'system layout', by generalizing the optimization techniques adopted in traditional theories, such as the Heuristic Layout Method (Liebman, 1967), Dynamic Programming (DP) (Argmar, Shamir and Spivak, 1973), Discrete Differential Dynamic Programming (DDDP) (Mays, Harry, Wenzwl and Liebman, 1976; Wen and Shih, 1983), Shortest Path Algorithm (Tekeli and Belkaya, 1986), Shortest Path Method (SPM) with Bounded Implicit Enumeration (BIE) (Liaw and Lin, 1990), and Integer Programming (IP) with Implicit Enumeration (IE) (Liaw, 1991, 1992, 1993).

To improve flaws in the local optimum problem for an optimal "system layout" model, Liaw and Weng (2003) developed the Sewer system Optimization Model for Layout \& Hydraulic Design (SSOM/LH) which considered problems of optimal "network layout" and "hydraulic design" simultaneously. This ensured that the solution had the best global optimum solution for a system layout with a 25-node (manhole) case study. Behind the SSOM/LH is to combine the Sewerage system Optimization Model (SSOM), to find an optimal hydraulic design for an urban sewer system. The application of a 0-1 Mixed Integer Programming (MIP) and a Bounded Implicit Enumeration (BIE) algorithm, allows SSOM to determine the size of each sewer pipe in a gravity sewer system, to meet the design objectives and to satisfy the hydraulic and technical constraints for some specific network layout in common use.

Recently, one of the Soft Computing, Genetic Algorithms (GA) has been popularly applied. Its robustness and efficiency seem to be an aid for researchers engaged in the environmental fields of analysis (McKinney and Lin, 1992, 1993, 1994). In this study, a GA will be applied to reestablish a combinatorial optimization model, GA/SSOM/LH, to help SSOM/LH to more efficiently generate some good alternate network layouts. This paper will describe the GA/SSOM/LH by the way of the SSOM/LH procedures.

\section{The Procedure of Optimal Sewer System Layout (SSOM/LH)}

The SSOM/LH is a combinatorial optimization model consists of a network layout generating procedure and the developed optimization hydraulic design module, SSOM, for a hydraulic design procedure. Both of the procedures perform as a screening role. Each generated layout must go through the hydraulic calculation, and then get a construction cost. Therefore, the optimal system layout procedure behind SSOM/LH can be described as follows.

\subsection{The Developed Hydraulic Design Optimization Mod- ule (SSOM)}

The SSOM has already been extensively discussed in another article (Weng and Liaw, 2003). Equation (1) shows the single objective equation of the SSOM with applied 0-1 mixed integer programming and constraints that meet the design criteria and regulatory standards at a minimal cost are represented by Equation (2-a) through Equation (2-d).

Objective:

$$
\begin{array}{r}
\text { Min } Z=\sum_{i=1}^{n} \sum_{j=1}^{m} \operatorname{Cost}\left(D_{i j}, S_{i j}, \operatorname{Hup}_{i}\right) X_{i j} \\
+\sum_{i=1}^{n} M H c\left(\operatorname{Hup}_{i}\right)+\sum_{i=1}^{n} P S_{i}\left(Q_{i}, H_{i}\right)
\end{array}
$$

Subject to:

$$
\begin{aligned}
& X_{i j}=0,1 ; i=1,2, \ldots, n, j=1,2, \ldots, m \\
& \sum_{i=1}^{n} X_{i j}=1 ; i=1,2, \ldots, n, j=1,2, \ldots, m \\
& H_{\min } \leq \operatorname{Hup}_{i} \leq H_{\max } ; i=1,2, \ldots, n \\
& H_{\min } \leq H d m_{i} \leq H_{\max } \\
& D_{i-1, j} \leq D_{i, j} ; i=1,2, \ldots, n, j=1,2, \ldots, m
\end{aligned}
$$

where $D_{i j}$ is the diameter of the $j$-th option of the $i$-th stage; $S_{i j}$ is the optimal slope to the certain diameter; Hup is the upstream pipe inverted or the manhole depth of $i$-th stage, meters; $H_{i}$ is the pumping head, meters; $Q_{i}$ is the pumping flow rate, CMD, if the $i$-th stage needed a pumping station; $H_{\text {min }}$ and $H_{\max }$ are the constrains of the minimum and maximum excavation depth, meter; $X_{i j}$ is the variety of the option between 0 and 1.

To minimize the total cost $Z$ including the cost of pipes, $D_{i j}$, of manholes, $\mathrm{MHc}\left(\mathrm{Hup}_{i}\right)$, and pumping stations, $P S i\left(Q_{i}\right.$, $H_{i}$ ), once a sewer network layout is found, the main function of the hydraulic design optimization module is to select the size and slope of the piping system and the number of pumping stations needed to meet the design criteria, with minimal construction cost to achieve the ultimate goal of costeffectiveness. Therefore, a hydraulic design problem can be treated as a serial multi-stage multi-option problem (Lin, 1990; and Liaw and Lin, 1991). The stages represent the sewer pipe network, and the options represent the pipe sizes available for each stage.

In Figure 1, Cases \#1 and \#2 show the response to a variety of construction modes, such as the open-cut method, trenchless technologies, and the pipe materials; Constrains \#1 and \#2 are the response to the limitations for each stage, such as passing through a fixed depth; $D_{i j}$ is the commercial standard diameter of the $j$-th option of the $i$-th stage; $S_{i j}$ is the 


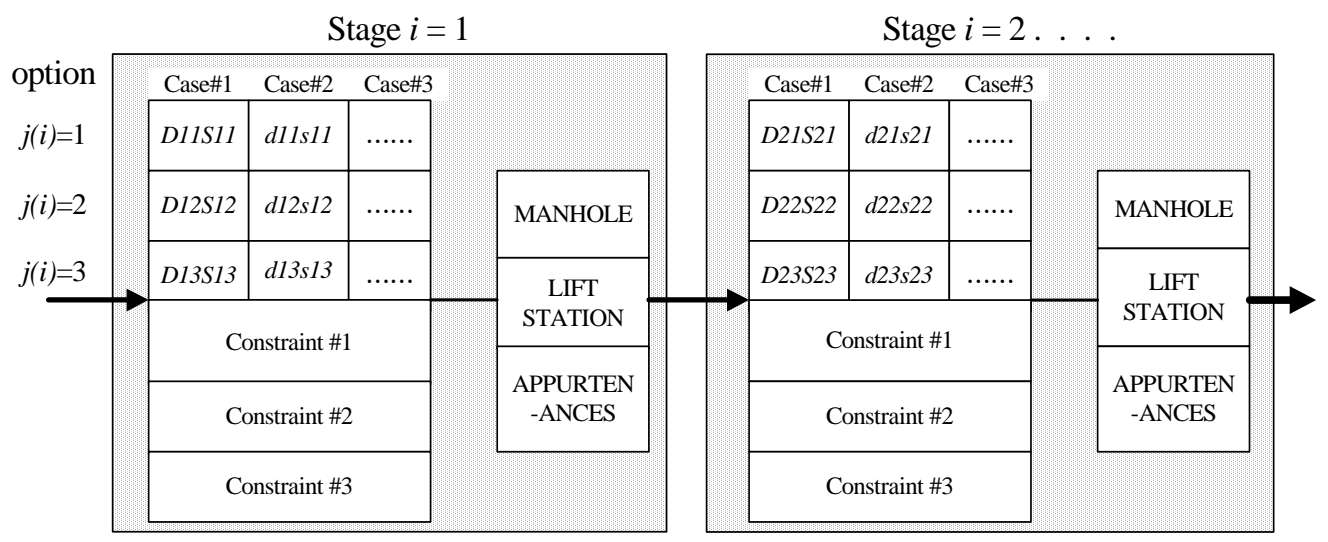

Figure 1. SSOM problem scheme of a serial multi-stage multi-option hydraulic design problem.

optimal slope for a specified diameter (here $i=1,2, \ldots, n, j=$ $1,2, \ldots, m)$. For each of the sewer pipes, different pipe sizes $\left(D_{i j}\right)$ are assigned as different options. Each pipe size is then associated with a minimal slope, $S_{i j}$, which was obtained by comparing the slopes associated with the minimal cover depth, the maximal flow velocity, the minimal flow velocity of partial flow, and the hydraulic force due to of gravity (Benson, 1985; Orth, 1986). Various construction modes and different piping material constraints are considered for each stage, such as passing through a fixed elevation, designating the site of a pumping station, or choosing the correct couple of diameter and slope for piping system. The SSOM problem scheme of a serial multi-stage multi-option hydraulic design problem is outlined in Figure 1.

In this configuration, the hydraulic design problem can be solved with discrete optimization techniques. The bounded implicit enumeration (BIE) algorithm is effective and efficient for solving serial multi-stage multi-option optimization problems (Chang and Liaw, 1990). The flow chart of the BIE algorithm used in the SSOM model is shown in Figure 2.

Figure 2 shows the tunnel jacking method that is not a trench dug along the piping line but only the digging of a shaft (pit), which is different from the open-cut method. The main design variables for the SSOM are the pipe sizes and shaft depths. For each stage, or sewer pipe, the minimal cost can be obtained by selecting the minimal pipe diameter and the minimal shaft depth for that stage. An actual sewer system construction case in Taipei was selected to compare the differences between the traditional design method and the SSOM. The comparison showed that the SSOM could find the most cost-effective design.

\subsection{The Optimal "Network Layout” Procedure}

The optimal "network layout" problem is similar to the optimal "hydraulic design" and can also be treated as a serial multi-stage multi-option optimization problem. In the problem scheme, the stages are marked by a manhole, where the flow direction in the network is different, and the options represent the two or more available flow directions for each manhole. In this configuration, the "network layout" can be solved with the traditional enumeration algorithm and will be not to omit the possible sewer system alternative. For example, a stage in the scheme represents a manhole with a two-flow-direction (TFD). Figure 3 shows TFD problem scheme for serial multi-stage multi-option network layout problems.

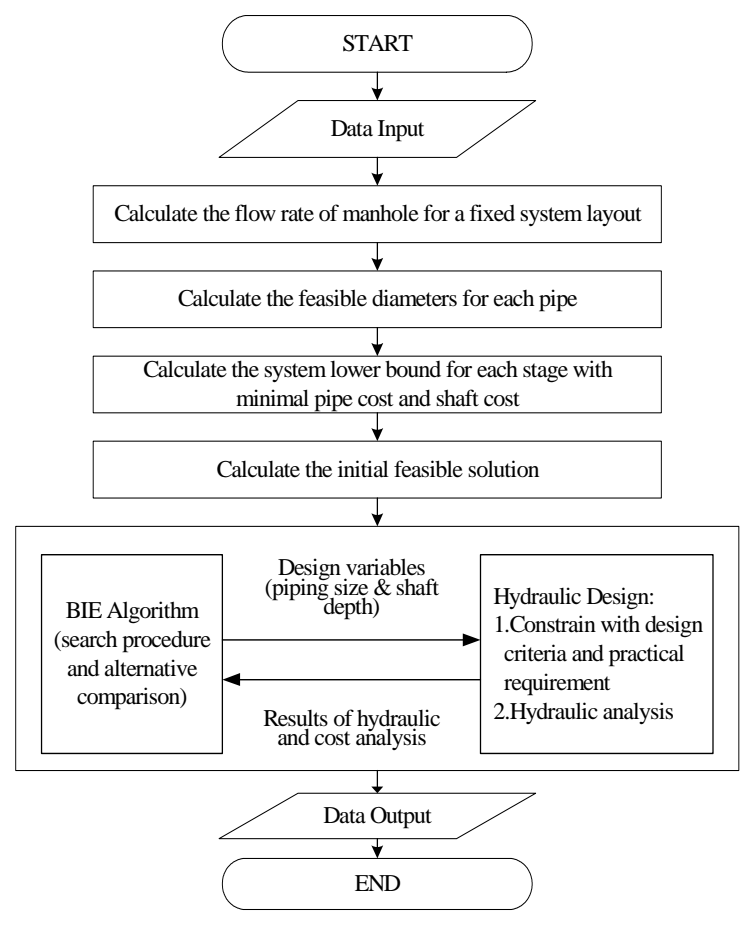

Figure 2. The flow chart of BIE algorithm used in the SSOM. 
In Figure 3, If only one flow direction can be chosen at each manhole or node, the option for a particular stage represents the flow direction or the sewer pipe that can be selected. Therefore, the procedure for solving sewer system layout problems is similar to that of other hydraulic design problems.

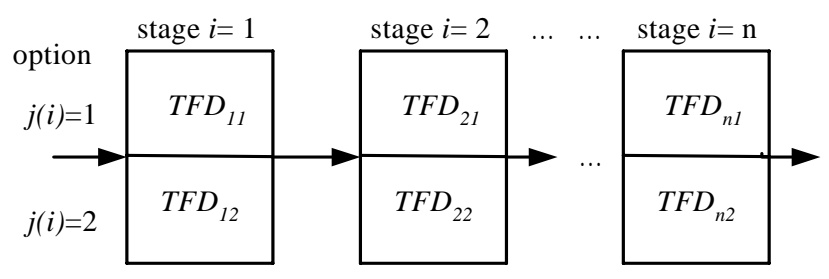

Figure 3. The TFD problem scheme for a serial multi-stage.

\subsection{The Performance of SSOM/LH Procedure}

Although, in this approach, the optimal solution obtained is undoubtedly the optimal design of a real sewer system layout. It is, however, very inefficient, almost impossible to solve, even with a mainframe or super computer. For a sewer system with $\mathrm{N}$ two-flow-direction (TFD) manholes, if the optimization of a hydraulic design problem requires one second of computation time, the time required to find the optimal solution will be $2^{\mathrm{N}}$ seconds. Thus, for only a moderate sized problem, it could take several years to find the optimal design with a large mainframe computer. If most of the TFD manholes can be fixed to have one flow direction before the problem solving process begins, the number of decision nodes will be greatly reduced. In this study, a heuristic approach based on engineering experience was used to eliminate the TFD nodes related to the population to be served, the street layout and the local topography of the planning area, so that the number of alternatives were reduced exponentially. This procedure makes it possible to solve sewer system design problems with Personal Computer.

\section{Applying GA to Sewer System Layout Optimiza- tion Model (GA/SSOM/LH)}

The optimization search procedure is hardly allowed to miss any possible sewer design alternatives during a feasible solutions screening search. It will be necessary but defective that to give the limitation of time-definiteness with applied for a large system analysis. The above-mentioned 25-node case study of the SSOM/LH has already successfully recommended a real optimal solution for a small sewer system layout (Weng, 2003), it will be time-consuming for the combinatorial algorithm, which is to combine the "network layout" and "hydraulic design" optimal procedure. Both of the optimal procedures can be viewed as a serial multi-stage multi-option optimization problem. The number of alternative combinations of the system increases exponentially as the numbers of "stages" are multiplied by the "options". In particular, for the optimization of an urban sewer system, the system components finding become too large thus leading to an N-P complete problem, a problem which is both N-P (verifiable in Nondeterministic Polynomial-time) (Wolfram Research, 2005).

Moreover, the robustness of the combinatorial models appear to be confined, since traditional algorithms are highly selective in problems they can solve and are limited in their scope of application. Therefore, an effective algorithm is the key to the establishment of the sewer system optimization model.

Research in recent years has adopted the GA to solve network system design optimization problems with an increasing trend (Simpson et al., 1994; Halhal et al., 1997; Savic and Walters, 1997; Lin et al., 1997; Pilar Montesinos et al., 1999; Dandy et al., 2001). The concept behind GA comes from the "Survival of the fittest in natural selection" of Darwin's evolutionary and was advanced by John Holland in 1970s. Different from traditional algorithms, the GA works with coding parameter string that can search from a population of points rather than a single point. It can use objective function information rather than derivatives or other auxiliary knowledge. It can use probabilistic transition rules rather than deterministic rules, and can surpass it is more traditional cousins in term of robustness. Therefore the GA can successfully solve discontinuous, non-differentiable, non-convex, and multiple peak function optimization problems.

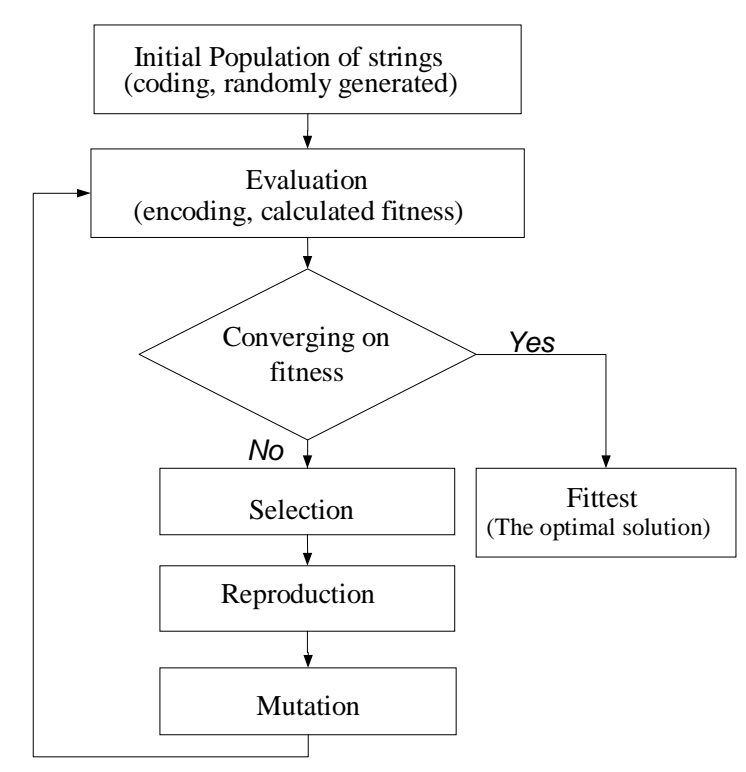

Figure 4. The flow chart of the simple GA evolutionary process.

Figure 4 shows the flow chart of a simple GA evolutionary process of the GA yields good results for many practical problems with any three basic operators: (1) reproduction (i.e. selection strings are copied according to fitness values); (2) crossover (i.e. members of reproduced strings are mated at random with each pair of strings undergoes crossing over); 


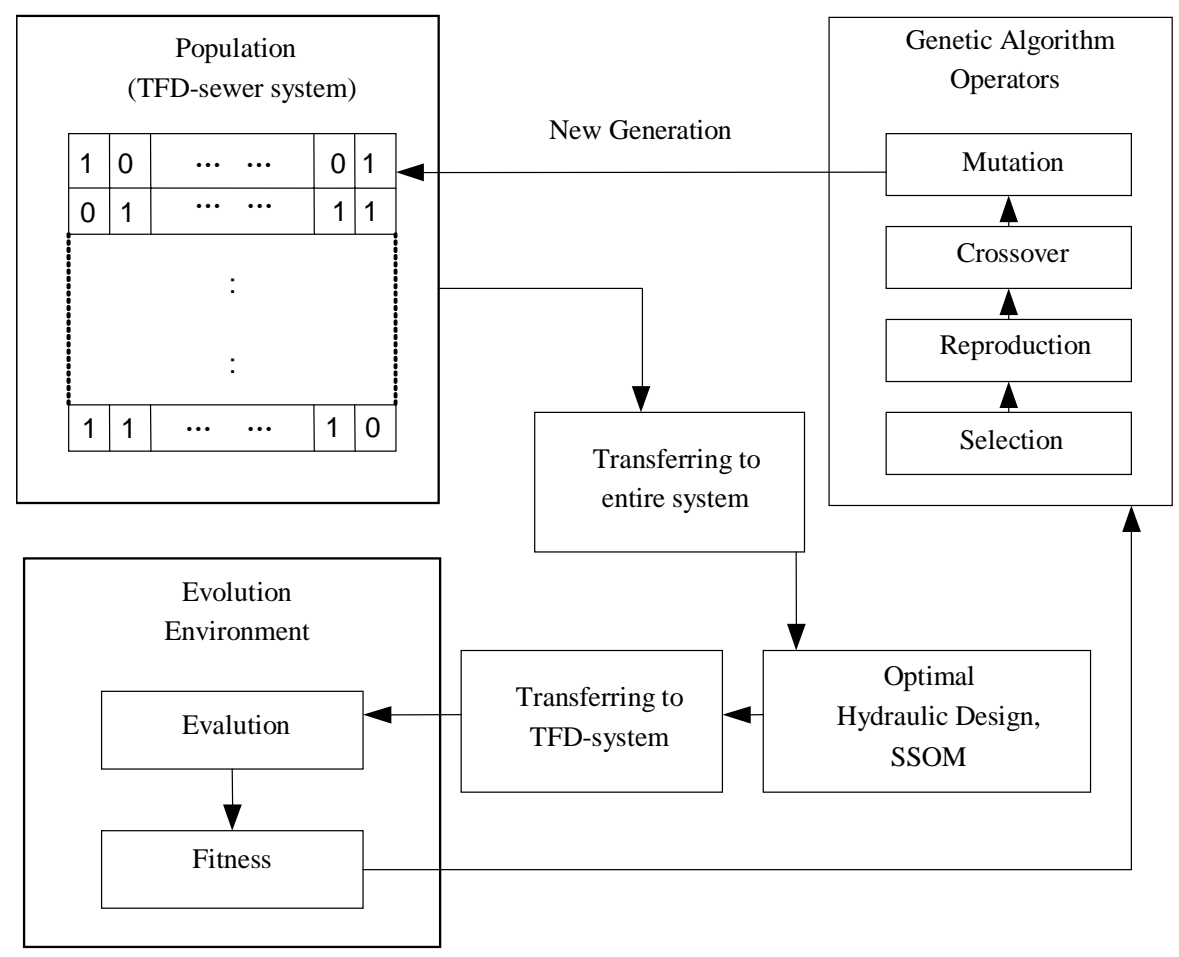

Figure 5. The scheme of the GA/SSOM/LH evolutionary process.

and (3) mutation (i.e. occasional random alterations that are hard to settle by most of traditional analytic and numerical optimized technologies) (Goldberg, 1989).

In this study, a GA will be applied to improve the algorithmic efficiency of the SSOM/LH and to reestablish a optimization model, GA/SSOM/LH, which combines the fundamental principles of the GA, to generate possible "network layout", based on SSOM that is a developing urban sewer system optimization module for checking the "hydraulic design”. The scheme of the GA/SSOM/LH constructed evolutionary process is shown as Figure 5.

Figure 5 includes three main blocks: (1) population selection for coding strings; (2) evolutionary environment for fitness evaluation; and (3) evolutionary computation of the three basic operators for the GA. In the population selection block process, the GA/SSOM/LH arranges "one chromosome" to represent "one network layout" and codes the genes of this chromosome with specific binary coding, to become a ' 0 ', ' 1 ' parameter string. Just by using these coded strings, a population was randomly selected to be the initial parent population, consisting of several chromosomes. Then, under the GA evolutionary environment process through the evolutionary computation process, a new generation could be produced to find some good alternate network layouts. Finally, the GA evolves into an offspring population by converging on the minimal cost fitness, to determine the optimal sewer system. In particular, the GA evolutionary process codes only unfixed node in the network layout. For example with the TFD-system, it is very important process to transfer the entire system layout of the SSOM hydraulic design at a forward transferring block (see Figure 5). Hence, the GA/SSOM/LH simulates genes. Different chromosomes are formulated evolve and generate 'new offspring' naturally, which allows the system to quickly converge to the actual optimization. In addition, the sewer network layout nodes and pipeline variables can be numbered easily. Therefore, the GA/SSOM/LH can generate alternate optimal network layouts more efficiently, eliminating inferior alternatives more economically in terms of execution time than the SSOM/LH.

\section{Case Study}

To test of the GA/SSOM/LH and compare with DDDP Model approach, we use a sewer system case that contains 73 nodes (manholes) with 48-TFD. The total nodes are laid out at the street corners. The TFD-nodes are indicated with a numerical arrowhead (see Figure 6.).

According to the GA/SSOM/LH evolutionary process, the 48-TFD network layout case can arrange for 'one chromosome', consisting of binary coding genes numbered " 0 or 1 ", to represent the two flow-direction options. Once the 48-TFD with a fixed flow direction are put into the network layout, a numerical coded string represents 'one network layout' of 48-TFD, indicating fixed flow direction in the net- 
work layout. Before into the GA/SSOM/LH process, there are some operating parameters in the GA program, i.e. the population size, the maximum number of generations, the probability of the jump mutation, the probability of uniform crossover, and etc, of a GA run. They are related to the convergence of the evolutionary process, should be set by a heuristic rule, to shorten the computation time. For this case study, GA algorithm parameters with the routine binary coding, were chosen as follows: the number of generations is 100 ; the number of populations is 10 ; the mating rate is 0.5 ; the mutation rate is 0.02 ; the objective of optimization is $1 /$ cost which fits the requirements for the "Maximizing" and "Nonnegative" fitness function, etc.

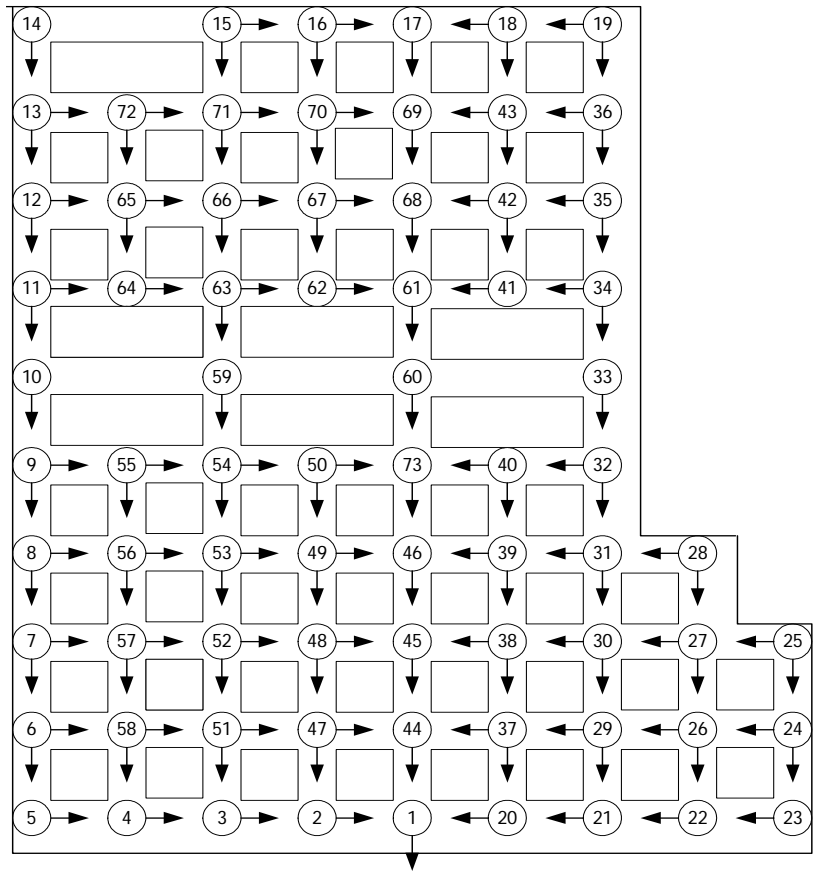

Figure 6. The street layout plan of 73 nodes with 48-TFD case.

To prove that the GA/SSOM/LH is running with the best fitness, and to ensure that the solution closest to the global optimization is observed in a 'fast' manner, a case with 73 nodes (manholes) and 48-TFD was used with the DDDP model to find the shortest sewer design (Shih, 1983). The result is a pipeline length of $3,882 \mathrm{~m}$, for 48 -TFD as shown as Figure 7.

In the case study, for a comparison in piping network hydraulic analysis with a case DDDP Model approach, the SSOM Model applies the same hydraulic design criteria. For example, using " $n=0.015$ ” as the same roughness coefficient of Manning's Formula, 0.9 and 10 meters as the minimum and maximum cover depth, 0.6 and 3 meters per second as the minimum and maximum velocity and etc. Also, we used the same cost function for the optimal hydraulic design procedure. The cost function is shown in Equation (3) for piping and in
Equation (4-a) through Equation (4-e) for manhole. For piping:

$C_{p}=0.051+0.383 D^{2}+0.0137 H^{2}$

For manhole:

$$
\begin{array}{lr}
C_{m}=0.725 H^{0.548}, & H \leq 0.25 m \\
C_{m}=0.8155 H^{0.579}, & 0.25 m<H \leq 0.8 m \\
C_{m}=1.1503 H^{0.484}, & 0.8 m<H \leq 1.2 m \\
C_{m}=1.7772 H^{0.355}, & 1.2 m<H \leq 1.65 m \\
C_{m}=2.1533 H^{0.313}, & 1.65 m \leq H
\end{array}
$$

where $C_{p}$ is the construction cost of piping, ten thousand $\mathrm{NT} \$ / \mathrm{m} ; C_{m}$ is the construction cost of manholes, ten thousand $\mathrm{NT} \$ / \mathrm{m} ; D$ is the pipe diameter, meter; $H$ is the excavation depth or manhole, meter.

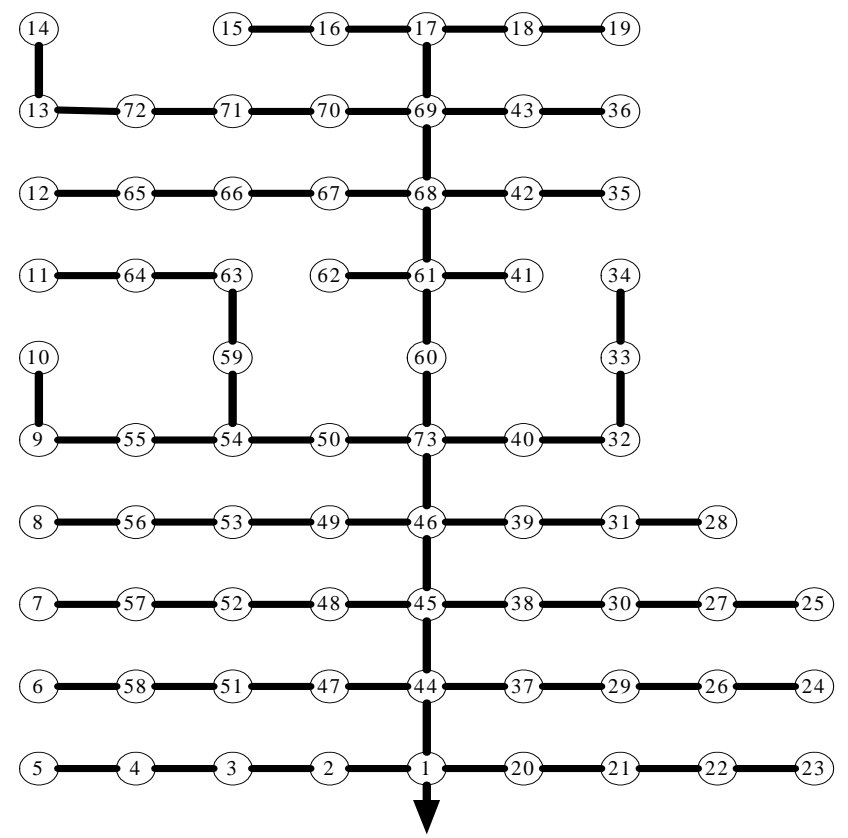

Figure 7. The sewer design from the DDDP model (48-TFD $3,882 \mathrm{~m})$.

\section{Results and Discussion}

(1) The case study was completed using a Personal Computer. The most cost-effective designs for a sewer system layout converge within the $93^{\text {rd }}$ generation, with a string parameter of ' 111111000001101111111111110111001100000 011111001'. A total 1,620,000 combinations was computed 
within 45 seconds. Each network layout, which was generated in the optimization procedure of the GA/SSOM/LH could be confirmed by the hydraulic design optimization module, SSOM, and the construction cost was calculated. Finally, the SSOM optimization hydraulic computation was printed out at the end of $100^{\text {th }}$ generation (see the hydraulic computation in Table 1 and the optimization plan in Figure 8).

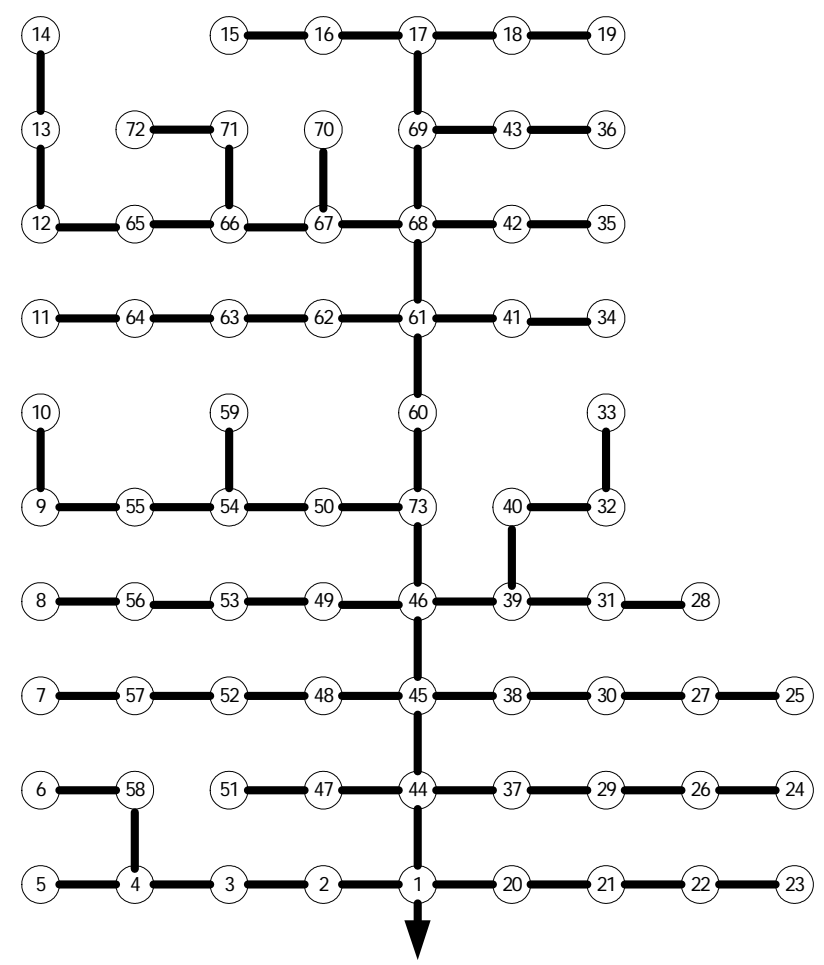

Figure 8. The GA/SSOM/LH optimal design plan (48-TFD 4,060 m).

(2) The results of the GA/SSOM/LH model at the end of the $100^{\text {th }}$ generation obtained the most cost-effective system layout, with a pipeline length of $4,060 \mathrm{~m}$ and a cost of NT\$ $1,297,820,000$, corresponding to the shortest pipeline length of $3,882 \mathrm{~m}$ with a cost of NT\$ $1,752,050,000$, generated by the DDDP model saving $30 \%$, in spite of the extra $178 \mathrm{~m}$ of pipeline. Therefore, the shortest sewer design was no guarantee that this would be a global optimum.

\section{Conclusions}

(1) The GA/SSOM/LH model established by this study used a full network system as a chromosome, which was coded in a binary parameter string numbered ' 0 ' and ' 1 '. The GA optimization method for offspring of greater fitness proved that it was able to obtain a more cost-effective optimal solution more quickly. The offspring population (secondary optimization designs) generated from the GA/SSOM/LH and its hydraulic analysis can be used as priority alternatives for reference.

(2) This study used a GA evolution for the structure of sewer system layout optimization, and proved its efficacy in utilizing the robustness of the GA and the convenience of solving more complicated pipeline system optimization problems with hybrid algorithms.

Acknowledgments. Many thanks to Mr. William Chung, the Project Manager of CTCI Corporation in Taiwan, who has contributed to the practical design parameter; and to Dr. Bi-Liang Lin, Assistant Prof. of Department of Civil Engineering in Ming-Hsin University of Science \& Technology of Taiwan, for his efforts at programming, during the development of the model.

\section{References}

Benson, R.E., (1985). Self-cleaning slope for partially full sewers. $J$. Environ. Eng. Div., ASCE, 111(6), 925-928.

Chang, S.Y. and Liaw, S.L. (1987). An efficient implicit enumeration algorithm for multistage systems, presentated at the TIMS/ORSA Joint National Meeting, New Orleans, LA, May 4-6.

Dandy, G.C. and Engelhardt, M. (2001). Optimal scheduling of pipe replacement using genetic algorithms. J. Water Resour. Plann. Manage., ASCE, 127(4), 214-223.

Goldberg, D.E. and The University of Alabama (1989). Genetic Algorithms in Search, Optimization, and Machine Learning, 1st Edition, Addison-Wesley Publishing Company, Inc., USA.

Gupta, A., Mehndiratta, S.L. and Khanna, P. (1983). Gravity waste water collection systems optimization, J. Environ. Eng. Div., ASCE, 109(5), 1195-1208.

Halhal, D., Walters G.A. and Savic, D.A. (1997). Water network rehabilitation with structured messy genetic algorithms. J. Water Resour. Plann. Manage., ASCE, 123(3), 137-146.

Hsu, C.L. (1984). Computer Aided Design for Large Sewerage Systems, A Thesis Submitted in Partial Fulfillment of the Requirements for the Degree of Master of Engineering, AIT.

Liaw, S.L. (1991). Optimization of Sewer System: System Layout, International Conference on Ccomputer Application in Water Resources, Tamkang University, 2, pp. 157-164.

Liaw, S.L. and Lin, B.L. (1991). Optimization of Sewer System: Hydraulic Design, International Conference on Computer Application in Water Resources, Tamkang University, 2, pp. 77-84.

Lin, B.L. (1990). An Optimization Model for Sewer System Layout and Design, A Thesis Submitted in Partial Fulfillment of the Requirements for the Degree of Master of Engineering, Tamkang University, Taiwan.

Mays, L.W. and Yen, B.C. (1975). Optimal cost design of branched sewer systems. Water Resour. Res., 11(1), 37-47.

McKinney, D.C. and Lin, M.D. (1994). Genetic algorithm solution of groundwater management models. Water Resour. Res., 30(6), 1897-1906.

Orth, H.M. (1986), Model-Based Design of Water Distribution and Sewage Systems, 1st Edition, John Wiley \& Sons, New York, USA.

Pilar, M., Adela, G.G. and Jose, L.A. (1999). Water distribution network optimization using a modified genetic algorithms. Water Resour. Res., 35(11), 3467-3473.

Savic, D.A. and Walters, G.A. (1997). Genetic algorithms for Least-cost design of water distribution networks. J. Water Resour. Plann. Manage., ASCE, 123(2), 67-77. 
Table 1. The GA/SSOM/LH Hydraulic Computation

\begin{tabular}{|c|c|c|c|c|c|c|c|c|c|c|}
\hline $\begin{array}{c}\text { Manhole } \\
\text { from }\end{array}$ & $\begin{array}{l}\text { No. } \\
\text { to }\end{array}$ & $\begin{array}{l}\text { Flowrate } \\
\text { (CMD) }\end{array}$ & $\begin{array}{c}\text { Length } \\
\text { (M) }\end{array}$ & $\begin{array}{l}\text { Slope } \\
(\%)\end{array}$ & $\begin{array}{c}\text { Diameter } \\
\text { (mm) }\end{array}$ & $\begin{array}{c}\text { UCE } \\
\text { up(M) }\end{array}$ & $\begin{array}{c}\text { DCE } \\
\operatorname{down}(\mathrm{M})\end{array}$ & $\begin{array}{l}\text { UGL } \\
\text { up(M) }\end{array}$ & $\begin{array}{c}\text { DGL } \\
\operatorname{down}(\mathrm{M})\end{array}$ & $\begin{array}{c}\text { Velocity } \\
\text { (M/S) }\end{array}$ \\
\hline 1 & 1 & 20884.4 & 0 & 0 & 0 & 0 & 0 & 4.81 & 4.81 & 0 \\
\hline 2 & 1 & 1908.8 & 80 & 0.60 & 0.2 & 2.1 & 1.62 & 4.79 & 4.81 & 0.7 \\
\hline 3 & 2 & 1523.9 & 80 & 0.30 & 0.2 & 2.34 & 2.1 & 4.79 & 4.79 & 0.6 \\
\hline 4 & 3 & 1260.6 & 80 & 0.35 & 0.2 & 2.62 & 2.34 & 4.74 & 4.79 & 0.6 \\
\hline 5 & 4 & 160.5 & 80 & 1.56 & 0.2 & 3.86 & 2.62 & 4.76 & 4.74 & 0.6 \\
\hline 6 & 58 & 301.1 & 80 & 0.98 & 0.2 & 4.08 & 3.29 & 4.98 & 4.83 & 0.6 \\
\hline 7 & 57 & 295 & 80 & 1.00 & 0.2 & 3.9 & 3.1 & 4.8 & 4.75 & 0.6 \\
\hline 8 & 56 & 307.1 & 80 & 0.97 & 0.2 & 3.9 & 3.13 & 4.8 & 5 & 0.6 \\
\hline 9 & 55 & 719.2 & 82 & 0.52 & 0.2 & 3.37 & 2.95 & 4.9 & 5.05 & 0.6 \\
\hline 10 & 9 & 396.4 & 78 & 0.80 & 0.2 & 4 & 3.37 & 4.9 & 4.9 & 0.6 \\
\hline 11 & 64 & 354.9 & 80 & 0.87 & 0.2 & 4 & 3.3 & 4.9 & 5 & 0.6 \\
\hline 12 & 65 & 1050.9 & 80 & 0.39 & 0.2 & 2.37 & 2.06 & 4.9 & 5.25 & 0.6 \\
\hline 13 & 12 & 786.6 & 120 & 0.49 & 0.2 & 2.96 & 2.37 & 5.07 & 4.9 & 0.6 \\
\hline 14 & 13 & 337 & 135 & 0.90 & 0.2 & 4.18 & 2.96 & 5.08 & 5.07 & 0.6 \\
\hline 15 & 16 & 496.1 & 80 & 0.68 & 0.2 & 4.32 & 3.77 & 5.22 & 5.25 & 0.6 \\
\hline 16 & 17 & 803.3 & 80 & 0.48 & 0.2 & 3.77 & 3.39 & 5.25 & 5.3 & 0.6 \\
\hline 17 & 69 & 1560.3 & 135 & 0.30 & 0.2 & 2.78 & 2.38 & 5.3 & 5.45 & 0.6 \\
\hline 18 & 17 & 496.1 & 80 & 0.68 & 0.2 & 3.32 & 2.78 & 5.35 & 5.3 & 0.6 \\
\hline 19 & 18 & 172.9 & 80 & 1.47 & 0.2 & 4.5 & 3.32 & 5.4 & 5.35 & 0.6 \\
\hline 20 & 1 & 1105.1 & 75 & 0.38 & 0.2 & 1.64 & 1.36 & 4.77 & 4.81 & 0.6 \\
\hline 21 & 20 & 691 & 80 & 0.54 & 0.2 & 2.07 & 1.64 & 4.77 & 4.77 & 0.6 \\
\hline 22 & 21 & 402.4 & 80 & 0.80 & 0.2 & 2.71 & 2.07 & 4.74 & 4.77 & 0.6 \\
\hline 23 & 22 & 129.2 & 60 & 1.82 & 0.2 & 3.8 & 2.71 & 4.7 & 4.74 & 0.6 \\
\hline 24 & 26 & 258.8 & 70 & 1.10 & 0.2 & 3.87 & 3.1 & 4.77 & 4.77 & 0.6 \\
\hline 25 & 27 & 129.2 & 78 & 1.82 & 0.2 & 3.87 & 2.45 & 4.77 & 4.86 & 0.6 \\
\hline 26 & 29 & 769.8 & 80 & 0.50 & 0.2 & 3.1 & 2.71 & 4.77 & 4.87 & 0.6 \\
\hline 27 & 30 & 530.9 & 90 & 0.65 & 0.2 & 2.45 & 1.86 & 4.86 & 4.93 & 0.6 \\
\hline 28 & 31 & 154.2 & 80 & 1.60 & 0.2 & 4.12 & 2.84 & 5.02 & 5.02 & 0.6 \\
\hline 29 & 37 & 1292.5 & 80 & 0.34 & 0.2 & 2.71 & 2.43 & 4.87 & 4.81 & 0.6 \\
\hline 30 & 38 & 1056.4 & 80 & 0.39 & 0.2 & 1.86 & 1.55 & 4.93 & 4.84 & 0.6 \\
\hline 31 & 39 & 588.4 & 80 & 0.60 & 0.2 & 2.84 & 2.36 & 5.02 & 5.01 & 0.6 \\
\hline 32 & 40 & 713.6 & 84 & 0.52 & 0.2 & 3.52 & 3.08 & 5.05 & 5.05 & 0.6 \\
\hline 33 & 32 & 390.5 & 78 & 0.81 & 0.2 & 4.15 & 3.52 & 5.05 & 5.05 & 0.6 \\
\hline 34 & 41 & 360.9 & 82 & 0.86 & 0.2 & 4.3 & 3.59 & 5.2 & 5.2 & 0.6 \\
\hline 35 & 42 & 313.1 & 80 & 0.95 & 0.2 & 4.35 & 3.59 & 5.25 & 5.3 & 0.6 \\
\hline 36 & 43 & 319.1 & 80 & 0.94 & 0.2 & 4.35 & 3.6 & 5.25 & 5.35 & 0.6 \\
\hline 37 & 44 & 1791.9 & 80 & 0.53 & 0.2 & 2.43 & 2.01 & 4.81 & 4.81 & 0.66 \\
\hline 38 & 45 & 1555.1 & 80 & 0.30 & 0.2 & 1.55 & 1.31 & 4.84 & 4.91 & 0.6 \\
\hline
\end{tabular}


(Table 1 continues)

\begin{tabular}{|c|c|c|c|c|c|c|c|c|c|c|}
\hline 39 & 46 & 2009.6 & 80 & 0.67 & 0.2 & 2.36 & 1.82 & 5.01 & 5.01 & 0.74 \\
\hline 40 & 39 & 991 & 100 & 0.41 & 0.2 & 3.08 & 2.66 & 5.05 & 5.01 & 0.6 \\
\hline 41 & 61 & 662.6 & 78 & 0.55 & 0.2 & 3.59 & 3.16 & 5.2 & 5.2 & 0.6 \\
\hline 42 & 68 & 886.6 & 80 & 0.45 & 0.2 & 3.59 & 3.23 & 5.3 & 5.3 & 0.6 \\
\hline 43 & 69 & 892.1 & 80 & 0.44 & 0.2 & 3.6 & 3.24 & 5.35 & 5.45 & 0.6 \\
\hline 44 & 1 & 19140 & 124 & 0.46 & 0.5 & -0.3 & -0.86 & 4.81 & 4.81 & 1.13 \\
\hline 45 & 44 & 17078.9 & 115 & 0.36 & 0.5 & 0.12 & -0.3 & 4.91 & 4.81 & 1.01 \\
\hline 46 & 45 & 14678 & 120 & 0.27 & 0.5 & 0.44 & 0.12 & 5.01 & 4.91 & 0.86 \\
\hline 47 & 44 & 1137.5 & 80 & 0.37 & 0.2 & 3.39 & 3.1 & 4.85 & 4.81 & 0.6 \\
\hline 48 & 45 & 1832.6 & 80 & 0.56 & 0.2 & 2.47 & 2.02 & 4.82 & 4.91 & 0.67 \\
\hline 49 & 46 & 1901.2 & 90 & 0.60 & 0.2 & 2.51 & 1.97 & 5.01 & 5.01 & 0.7 \\
\hline 50 & 73 & 2475 & 90 & 1.01 & 0.2 & 1.92 & 1.01 & 5.1 & 5.05 & 0.91 \\
\hline 51 & 47 & 582.7 & 80 & 0.61 & 0.2 & 3.88 & 3.39 & 4.78 & 4.85 & 0.6 \\
\hline 52 & 48 & 1345.5 & 80 & 0.33 & 0.2 & 2.73 & 2.47 & 4.81 & 4.82 & 0.6 \\
\hline 53 & 49 & 1395.6 & 80 & 0.32 & 0.2 & 2.76 & 2.51 & 5 & 5.01 & 0.6 \\
\hline 54 & 50 & 2229.2 & 80 & 0.82 & 0.2 & 2.58 & 1.92 & 5.1 & 5.1 & 0.82 \\
\hline 55 & 54 & 991 & 90 & 0.41 & 0.2 & 2.95 & 2.58 & 5.05 & 5.1 & 0.6 \\
\hline 56 & 53 & 867.2 & 80 & 0.45 & 0.2 & 3.13 & 2.76 & 5 & 5 & 0.6 \\
\hline 57 & 52 & 836.7 & 80 & 0.47 & 0.2 & 3.1 & 2.73 & 4.75 & 4.81 & 0.6 \\
\hline 58 & 4 & 853.4 & 124 & 0.46 & 0.2 & 3.29 & 2.72 & 4.83 & 4.74 & 0.6 \\
\hline 59 & 54 & 758.6 & 78 & 0.50 & 0.2 & 4.1 & 3.71 & 5 & 5.1 & 0.6 \\
\hline 60 & 73 & 9659.1 & 78 & 0.20 & 0.45 & 0.82 & 0.66 & 5.1 & 5.05 & 0.7 \\
\hline 61 & 60 & 9174.2 & 80 & 0.18 & 0.45 & 0.97 & 0.82 & 5.2 & 5.1 & 0.67 \\
\hline 62 & 61 & 1529.1 & 80 & 0.30 & 0.2 & 2.58 & 2.34 & 5 & 5.2 & 0.6 \\
\hline 63 & 62 & 1255.3 & 80 & 0.35 & 0.2 & 2.85 & 2.58 & 5.1 & 5 & 0.6 \\
\hline 64 & 63 & 645.6 & 80 & 0.56 & 0.2 & 3.3 & 2.85 & 5 & 5.1 & 0.6 \\
\hline 65 & 66 & 1570.7 & 80 & 0.29 & 0.2 & 2.06 & 1.82 & 5.25 & 5.4 & 0.6 \\
\hline 66 & 67 & 2942.3 & 80 & 0.44 & 0.25 & 1.82 & 1.47 & 5.4 & 5.35 & 0.69 \\
\hline 67 & 68 & 3881.2 & 80 & 0.29 & 0.3 & 1.47 & 1.24 & 5.35 & 5.3 & 0.64 \\
\hline 68 & 61 & 7213.6 & 130 & 0.21 & 0.4 & 1.24 & 0.97 & 5.3 & 5.2 & 0.66 \\
\hline 69 & 68 & 2818.2 & 120 & 0.40 & 0.25 & 2.38 & 1.9 & 5.45 & 5.3 & 0.66 \\
\hline 70 & 67 & 617.1 & 120 & 0.58 & 0.2 & 4.55 & 3.85 & 5.45 & 5.35 & 0.6 \\
\hline 71 & 66 & 1034.6 & 120 & 0.40 & 0.2 & 3.51 & 3.04 & 5.4 & 5.4 & 0.6 \\
\hline 72 & 71 & 301.1 & 80 & 0.98 & 0.2 & 4.3 & 3.51 & 5.2 & 5.4 & 0.6 \\
\hline 73 & 46 & 11797.7 & 124 & 0.17 & 0.5 & 0.66 & 0.44 & 5.05 & 5.01 & 0.69 \\
\hline
\end{tabular}


Shih, C.S. (1983). Application of DDDP in Routing of Sewer System and Selecting of Pipe Size and Excavation Depth, A Thesis Submitted in Partial Fulfillment of the Requirements for the Degree of Master of Engineering, Cheng-Kung University, Taiwan.

Simpson, A.R., Dandy, G.C. and Murphy, L.J. (1994). Genetic algorithms compared to other techniques for pipe optimization. $J$. Water Resour. Plann. Manage., ASCE, 120(4), 423-443.

Swamee, P.K. (2001). Design of sewer line. J. Environ. Eng., 127(9), 776-781.

Tekeli, T. and Belkaya, H. (1986). Computerized layout generation for sanitary sewers. J. Environ. Eng. Div., ASCE, 112(4), 500-515.
Weng, H.T. and Liaw, S.L. (2003). An optimization model for sewer hydraulic design (SSOM) base on novel trenchless technology, in Proc. IWA Asia-Pacific Regional Conference, Bangkok, Thailand, Oct. 19-23.

Weng, H.T. and Liaw, S.L. (2003). The study of applying enumeration algorithm to a sewer system layout optimization model (SSOM/LH), in Proc. 16th Environmental Planning and Management Conference of CIEnvE, Taiwan, Nov. 28-29.

Wolfram Research (2005). MathWorld ${ }^{\mathrm{TM}}$ the web's most complete mathematical resource created and maintained by Eric W. Weisstein, http://mathworld.wolfram.com/ (accessed Feb. 18, 2005). 